\title{
Managing inventory and production capacity in start-up firms
}

\author{
Thomas W. Archibald \\ University of Edinburgh Business School, Edinburgh, UK \\ Edgar Possani \\ Department of Mathematics, Instituto Tecnologico Autonomo de Mexico ITAM, Mexico City, Mexico \\ Lyn C. Thomas \\ School of Management, University of Southampton, Southampton, UK
}

\begin{abstract}
We consider the problem of managing inventory and production capacity in a startup manufacturing firm with the objective of maximising the probability of the firm surviving as well as the more common objective of maximising profit. Using Markov decision process models, we characterise and compare the form of optimal policies under the two objectives. This analysis shows the importance of coordination in the management of inventory and production capacity. The analysis also reveals that a start-up firm seeking to maximise its chance of survival will often choose to keep production capacity significantly below the profit maximising level for a considerable time. This insight helps us to explain the seemingly cautious policies adopted by a real start-up manufacturing firm.
\end{abstract}

Keywords: start-up firms; inventory; production capacity; stochastic modelling; Markov decision process

\section{Introduction}

Start-up firms make an important contribution to the success of a country's economy by creating jobs and increasing competition and innovation. However, such firms face a high risk of failure during the start-up phase - more than $50 \%$ of manufacturing firms fail in the first four years in the US (Statistic Brain, 2013). Hence, there is a strong need for models that provide insight into the problems faced by start-up firms and help to identify strategies that ensure the long-term survival of such firms. Two decisions that have a significant effect on the chance of long-term survival of a start-up manufacturing firm are the choice of production capacity, both initially and in the early periods, and the level of capital (or borrowing) available to the firm. This paper investigates the characteristics of optimal policies for start-up manufacturing firms by modelling their production capacity decisions and inventory strategy during the start-up phase. Part of the motivation for developing and analysing the model in this paper was the case of a start-up manufacturing firm whose management believed that capacity expansion was too risky even though the firm often struggled to keep up with demand. Our aim was to investigate whether this attitude could be explained if one assumed the firm was really more interested in survival rather than profit maximisation.

The model developed is intended to help inform decisions taken by an entrepreneur who seeks to borrow money to start a manufacturing firm. During the start-up phase, the entrepreneur aims to establish a reputation which will provide a platform for subsequent growth of the firm. Therefore, the objective can be thought of as maximizing the probability of survival. In the model, the firm is said to fail if it has insufficient funds to meet its overhead costs. The overhead 
costs include the interest on the money borrowed to start the firm and the remuneration of the entrepreneur. The overhead costs will typically also include employee wages, equipment lease charges and rent for premises.

Due to the limited capital available to the firm, the optimal survival policy must balance investment of capital in production capacity and inventory with reserves of capital to cover overhead costs in cases of low demand. We contrast this situation with that of a well-established manufacturing firm which has almost no constraint on working capital and so can concentrate on profit maximisation. Specifically, the model provides insight on the following decisions:

- The optimal production capacity and inventory strategies for the firm.

- How much the entrepreneur should borrow given any particular risk threshold.

- The optimal initial production capacity for any particular level of borrowing.

- How to detect the end of the start-up phase.

We recognise that the survival of start-up firms depends on more than just their capacity and inventory decisions. The portfolio of products, the marketing strategy initiated, the relationship with suppliers and financial backers are also vital. However, one has to understand the impact of the separate components before one can deal with the complete situation. Moreover, in the conclusions we point out how the capacity model can be reinterpreted to reflect the marketing decisions that have to be made.

Management science models of manufacturing problems almost always include the objective of optimising the cost or profit to the firm (Silver et al., 1998). Archibald et al. (2002) suggest that such models are not suitable for start-up firms whose working capital is generally limited. They were the first to suggest that start-up firms should focus on maximising the probability of survival rather than optimising cost or profit. They look at this in the case of a multi-period stock control problem. The work concentrates on inventory strategy and assumes production capacity of the firm is fixed. There is little research on joint production and financial decisions for start-up manufacturing firms. The idea of start-up firms seeking to maximise survival probability has been extended to other inventory and production problems (Possani et al., 2003; Archibald et al., 2007; Swinney et al., 2011). The first two look at infinite planning horizons while the latter uses a one period model to look at the competition between start-up and established firms.

There has been a lot more research on joint production and financial decisions in the last decade. Betts and Johnston (2001) also focus on the inventory strategy of a manufacturing firm with limited capital. They compare the optimal strategy for their model with those of traditional modelling approaches to inventory management in a deterministic setting. Babich and Sobel (2004) develop a discrete time model of start-up firms with the objective of maximising expected discounted proceeds from initial public offerings. Buzacott and Zhang (2004) investigate the impact of asset-based loans and interest rates on the inventory decisions of cash-constrained firms. Unlike the problem analysed in this paper, the production capacity is not considered variable. The review by van Mieghem (2003) explains how risk aversion is incorporated within capacity planning models using corporate finance approaches. However, these approaches do 
not consider the probability of the firm failing as a measure of risk. Li et al. (2013) use expected present value of the firm as their criterion to make decisions about what short term loans to take each period, how much to produce, and what dividends, if any, to pay. Tanrisever et al. (2012) look at a two period model with production and investment decisions. They find that it is optimal to select a production level in the first period which is less than the profit maximising level if the start-up firm's objective is to maximise survival. This concurs with the conclusions of the model developed in this paper.

Jammernegg and Reiner (2007) note that models of inventory systems often assume fixed production capacity and argue that more attention should be given to coordinated inventory and production capacity management. The limited research in this area generally uses models that seek to optimise profit or cost with no restriction on borrowing. Therefore these studies consider applications that are very different from the focus of this paper. Cantamessa and Valentini (2000) use mixed-integer linear programming to find the optimal initial production capacity and inventory strategy for a manufacturing firm. Their model differs significantly from ours because it does not allow the firm any control over subsequent changes in production capacity and assumes future demand is known. Chan et al. (2006) use a Markov decision process model to investigate pricing, production and inventory decisions in a manufacturing context. Demand is uncertain, but unlike our model the changes to the production level incur no cost. Terwiesch and Bohn (2001) develop a deterministic dynamic programming model of production ramp-up in which the firm can choose to lower production capacity in the short-term in order to free up time for training and so increase future production capacity. Dellaert and de Kok (2004) consider an inventory problem in which production capacity can be increased by hiring a temporary workforce. Their model does not consider long-term changes to production capacity. Mincsovics et al. (2009) consider a similar problem and focus on the time required to make temporary changes to the production capacity. Çınar and Güllü (2012) analyse an inventory problem in which production can be outsourced as a hedge against uncertainty in demand and production capacity.

The model we introduce in this paper has a cost for increasing or decreasing the capacity of the system, and so has some similarities with the work of Sobel $(1969,1970,1971)$. Angelus and Porteus (2002) consider a similar cost structure in a model of the management of production capacity over the life-cycle of a make-to-stock product. Under the objective of minimising expected cost, it is shown that the optimal production capacity plan can be characterised by a target interval for each inventory level in each period. In each period, production capacity should be changed by the smallest amount necessary to bring the level into the relevant interval. However, our is the first paper to consider such a cost structure in the survival maximisation context.

In section 2 we develop Markov decision process models of start-up and well-established manufacturing firms who must make decisions about capital investment in inventory and production capacity in the face of uncertain demand. We also characterise the optimal strategy for the model of well-established firms. In section 3 we derive properties of the optimal strategy for a start-up manufacturing firm with the objective of maximising its survival probability. 


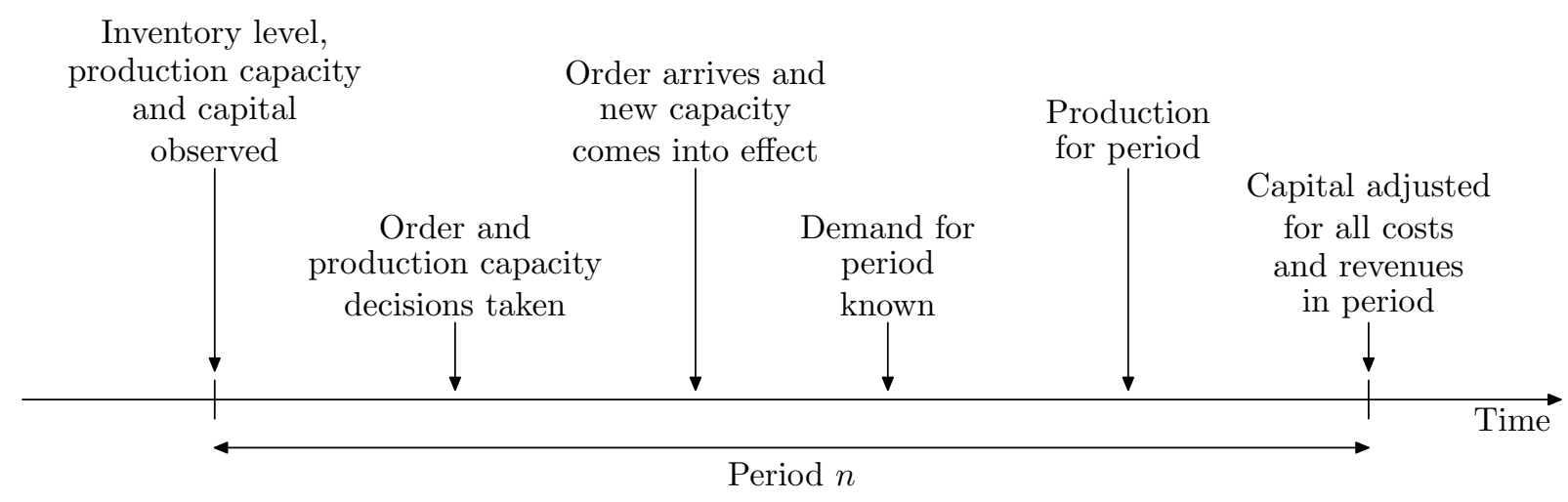

Figure 1: Time lines showing the sequence of events in one period.

Addressing the problem that motivated this research, in section 4 we describe the application of the model to the situation facing a real start-up manufacturing firm. Finally in section 5 we present our conclusions.

\section{Models of manufacturing firms}

Consider a firm selling, at price $S$, one type of product that it manufactures to order from a component (or group of components) it purchases at a cost $C$. Before the demand for each period is known, the firm has the opportunity to order components and change the production capacity. Both actions have an immediate effect. (Although not reported in this paper, we have obtained similar results from the analysis of a model in which the lead times for ordering components and changing production capacity are both one period. The production capacity is determined by equipment, number of staff and staff training.) Any change to the production capacity (up or down) incurs a one-off charge of $R$ per unit of production capacity and takes effect after $L_{r}$ periods. Each period the firm has to meet a fixed overhead cost $H$ plus a variable overhead cost of $r$ per unit of production capacity. The one-off charge $R$ covers, for example, the costs of acquiring or disposing of equipment and reorganising the workforce, while the recurrent cost $r$ models changes in the cost of finance, labour etc. The results of the paper hold if one has different costs $R_{1}$ for increasing production and $R_{2}$ for decreasing production, but for ease of understanding we will use $R$ for both costs hereafter. Although there is no direct inventory cost in the model, it is assumed that the cost of capital is included in the fixed overhead cost. Possani et al. (2003) show that introducing a direct inventory cost to a simpler model does not alter the results, only the analysis. The demand for the product each period is an independent identically distributed random variable. The maximum possible production capacity, and hence, the maximum demand that can be satisfied in a period, is $M$. Demand that cannot be satisfied in the period it arises is lost. For $0 \leq d<M$, let $p(d)$ denote the probability that there is a demand for $d$ items in a period and let $p(M)$ denote the probability that there is a demand for at least $M$ items in a period. Figure 1 shows the time line for the events in one period for this problem.

It is assumed that a start-up firm has a limited amount of working capital and has the 
objective of maximising its chance of survival. As the firm manufactures the product to order, an inventory of manufactured products will never be carried over from one period into the next. This situation may arise if, for example, storage of the product is impractical or the exact specification of the product is determined by the customer. Hence, the state of the firm at the start of a period is described by the number of components in stock, the production capacity and the amount of available capital. Each period the firm must decide the order quantity, $k$, and the new production capacity, $j^{\prime}$. Let $q(n, i, j, x)$ be the maximum probability that the firm survives for $n$ periods given it currently has $i$ components in stock, $j$ units of production capacity and $x$ units of available capital. We assume that the firm survives an interval of $n$ periods if and only if the amount of available capital is non-negative at the start and end of every period in the interval. This is reflected in the boundary conditions $q(0, i, j, x)=1$ if $x \geq 0$ and $q(n, i, j, x)=0$ if $x<0$ for all $n \geq 0$. Following the approach of Archibald et al. (2002), the problem can be formulated as a Markov decision process.

As the length of the start-up phase is not easily determined in advance, it is interesting to consider how the length of the planning horizon affects the optimal survival policy. Figure 2 shows the optimal first period decisions as a function of the length of the planning horizon for the motivating example considered in this paper. As the length of the planning horizon increases, the optimal inventory and production capacity decisions quickly converge to the optimal levels for an infinite horizon planning horizon - the graph follows a horizontal line when the planning horizon has more than 17 periods. As one might expect, the optimal decisions are more conservative when the planning horizon is very short and may be zero if one can survive the planning horizon on existing capital. In such cases investment in production capacity is less attractive, because there is little time in which to generate a return on the investment. We conclude that analysis of infinite horizon models can provide insight about the inventory and production capacity decisions in a start-up firm except, perhaps, for very short planning horizons. We focus our attention on infinite horizon models for the remainder of the paper.

The infinite horizon model of the start-up firm considers $q(i, j, x)=\lim _{n \rightarrow \infty} q(n, i, j, x)$ which can be interpreted as the maximum probability that the firm survives in the long-run given that it currently has $i$ components in stock, $j$ units of production capacity and $x$ units of available capital. It is easy to see that $q(n, i, j, x)$ is a bounded, monotonic sequence in $n$, as the probability of survival must lie between 0 and 1 . Moreover, in any scenario in which the firm survives $n$ periods, it must first survive $n-1$ periods, so $q(n, i, j, x)$ is non-increasing in $n$. It follows that the limit $q(i, j, x)$ exists. The firm is said to fail if the available capital is ever negative which corresponds to boundary conditions $q(i, j, x)=0$ if $x<0$.

As there is no fixed order cost and the lead time for ordering is zero, it is easy to show that it is never necessary to store, order or produce more than $M$ items in a period. Assuming that all revenues and costs can be expressed as multiples of a common unit, the problem can be formulated as a finite horizon Markov decision process with a countable state space and finite action space. The state space is given by $\{(i, j, x): 0 \leq i, j \leq M, x$ integer $\}$ and the action space is given by $\left\{\left(k, j^{\prime}\right): 0 \leq k, j^{\prime} \leq M\right\}$. For $x \geq 0$, the optimality equation for the model under the 


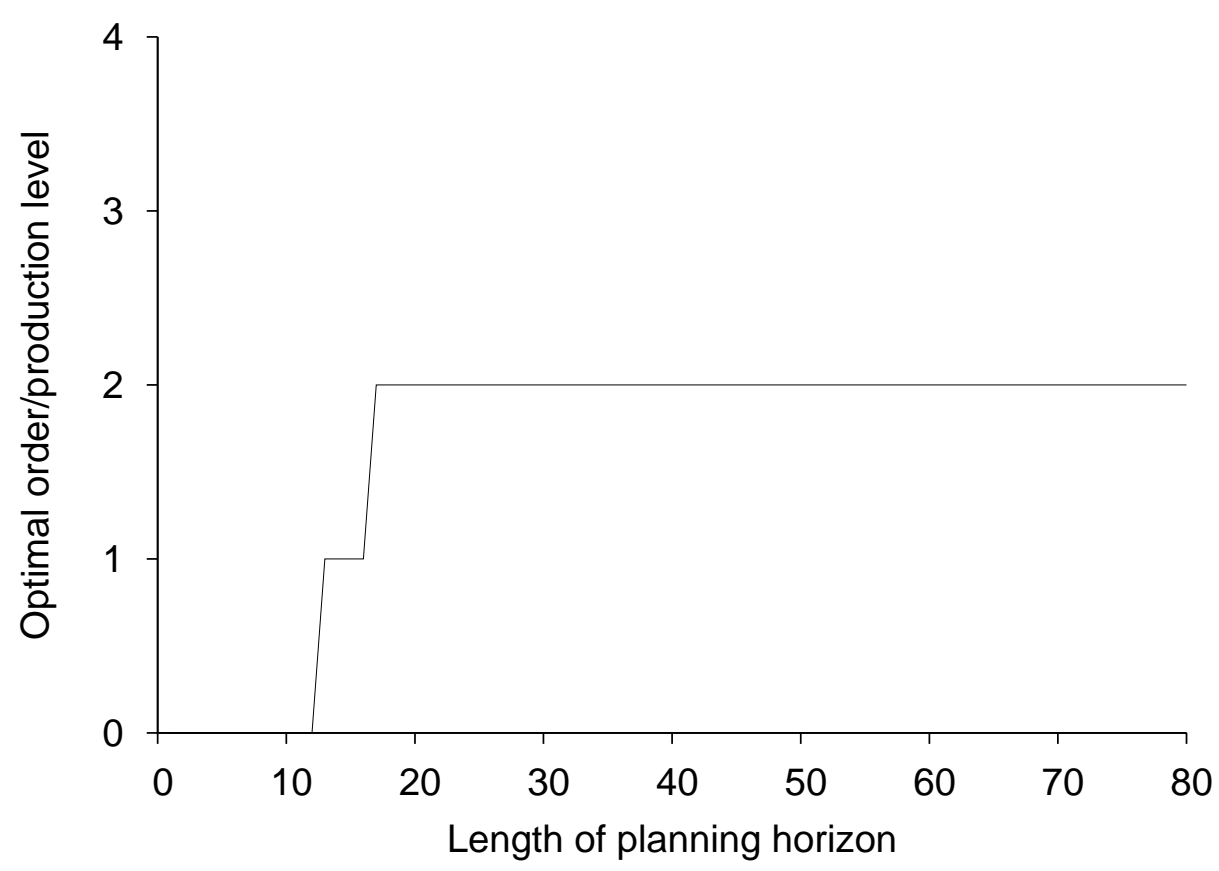

Figure 2: Effect of planning horizon on optimal survival policy for initial capital $X=45$. Initially the inventory level and production capacity are both $0 . S=15, C=9, H=4, R=5$, $r=3$ and $M=12$.

above assumptions is as follows.

$$
\begin{aligned}
q(i, j, x)=\max _{k, j^{\prime}}\left\{\sum _ { d = 0 } ^ { M } p ( d ) q \left(i+k-\min \left(i+k, j^{\prime}, d\right), j^{\prime},\right.\right. \\
\left.\left.x+\min \left(i+k, j^{\prime}, d\right) S-k C-\left|j-j^{\prime}\right| R-H-j^{\prime} r\right)\right\}
\end{aligned}
$$

This follows since sales in a period are limited by the available inventory $(i+k)$, the production capacity $\left(j^{\prime}\right)$ and the demand $(d)$. Hence the sales during the period will be $\min \left(i+k, j^{\prime}, d\right)$ and the number of components in stock at the beginning of the next period will be $i+k-$ $\min \left(i+k, j^{\prime}, d\right)$. The capital available to the firm becomes the original capital plus the income from sales minus the sum of the cost of the components ordered, the one off cost of changing the production level, the fixed overhead cost and the cost of running the production unit at the new level, i.e.

$$
x+\min \left(i+k, j^{\prime}, d\right) S-k C-\left|j-j^{\prime}\right| R-H-j^{\prime} r .
$$

One important consideration for a start-up firm is the maximum capital available to it initially — be it in the form of equity, venture capital or bank loans. Once this together with any revenue generated has been exhausted the firm will be forced to cease to trade. If a start-up firm has initial capital $X$, then its objective is to maximise $q(0,0, X)$.

It is assumed that for an established firm, there is no practical constraint on the amount of capital available and that the objective is to maximise the long-run average profit per period. The state of the firm at the start of a period is described by the number of components in stock and the production capacity. Each period the firm must decide the order quantity, $k$, and 
the new production capacity, $j^{\prime}$. Standard results for average reward Markov decision processes (Puterman, 1994) can be applied as follows. Let $g$ be the maximum average reward per period and let $v(i, j)$ be the bias term of starting with $i$ components in stock and $j$ units of production capacity. The optimality equation of the Markov decision process model of the firm under the above assumptions is as follows.

$$
\begin{aligned}
g+v(i, j)=\max _{k, j^{\prime}}\left\{\sum_{d=0}^{M} p(d)(\right. & \min \left(i+k, j^{\prime}, d\right) S-k C-\left|j-j^{\prime}\right| R \\
& \left.\left.-H-j^{\prime} r+v\left(i+k-\min \left(i+k, j^{\prime}, d\right), j^{\prime}\right)\right)\right\}
\end{aligned}
$$

The model has finite state space $\{(i, j): 0 \leq i, j \leq M\}$ and finite action space $\left\{\left(k, j^{\prime}\right): 0 \leq\right.$ $\left.k, j^{\prime} \leq M\right\}$. Let $k_{q}(i, j)$ and $k_{r}(i, j)$ be the optimal order quantity and new production capacity respectively.

In the following theorem we show that the maximum long-run average profit per period is achieved by striking a balance between the marginal increases in the overhead costs per period and the expected profit from sales per period as the production capacity increases.

Theorem 1. Define

$$
j^{*}=\min \left\{d^{*} \mid \sum_{d>d^{*}}(S-C) p(d)<r\right\} .
$$

The optimal average reward, the optimal bias terms and an optimal policy are given by the following.

$$
\begin{aligned}
& g=(S-C) \sum_{d=0}^{M} p(d) \min \left(j^{*}, d\right)-H-j^{*} r \\
& v(i, j)=i C-\left|j-j^{*}\right| R \\
& k_{r}(i, j)=j^{*} \\
& k_{q}(i, j)=\max \left(0, j^{*}-i\right)
\end{aligned}
$$

Proof. It is easy to verify by substitution that the given values for $g$ and $v(i, j)$ satisfy the optimality equations for the stated policies. Now apply the policy improvement step of policy iteration (Puterman, 1994) to verify that the policies are optimal. Further details are provided in the appendix.

\section{Properties of the optimal survival strategy for a start-up firm}

In this section we establish some important properties of the inventory and production capacity decisions that maximise the survival probability for a start-up manufacturing firm under the assumptions of model (1) above.

The first result provides insight about the relationship between optimal inventory and production capacity decisions for the survival model. What it shows is that on the "way up", i.e. when the firm is seeking to increase production, production and inventory levels are the same. On the "way down", i.e. when production capacity is too high for the capital available, the firm cuts the production level immediately, which disconnects production from the inventory level, and does not try to bring both down hand in hand, as that will take too long. The "on 
the way down" conditions occur either when the initial production level is too high or when the capital available falls due to a run of poor demand.

Theorem 2. In state $(i, j, x)$ there exist optimal ordering and production capacity decisions, $k$ and $j^{\prime}$ respectively, satisfying:

(i) either $k=0$ or $i+k \leq j^{\prime}$;

(ii) either $j^{\prime} \leq j$ or $i+k \geq j^{\prime}$.

Proof. See appendix.

From Theorem 2 (ii), if $j^{\prime}>j$ then either $i+k>j^{\prime}$ or $i+k=j^{\prime}$. Then, from Theorem 2 (i), it follows that if $j^{\prime}>j$ then either $k=0$ or $i+k=j^{\prime}$. Thus, if the production capacity increases, then either the firm does not order or it orders to make the inventory level equal to the level of capacity. Similarly, if $k>0$ then either $j^{\prime} \leq j$ or $i+k=j^{\prime}$. Hence, if the firm orders components and increases production capacity, then it orders to make the inventory level equal to the level of capacity. Furthermore, the following is true: either $i+k=j^{\prime}$ or $k=0$ or $j^{\prime} \leq j$. That is, if the new level of capacity is different from the new level of inventory, then either no inventory was ordered or the capacity was not increased.

This analysis raises questions about the importance of independent inventory and production capacity decisions to the survival of the firm. In the numerical examples which we will discuss in more detail later, situations arise where the optimal survival policy does not set the production capacity equal to the inventory level (allowing for any order placed) at the beginning of a period. Figure 3 illustrates two such situations which come from the motivating example with particular initial capacity and inventory levels. In Figure 3a, even though the initial inventory level is 7 , the production capacity chosen never exceeds 4 regardless of the initial level of production capacity. In Figure 3b we see that, even though the initial production capacity and inventory level are both 10, when the initial capital available to the firm is less than 400, the optimal decision is almost always to reduce the production capacity and so create a situation where production capacity is less than inventory level. In general, when the optimal survival policy sets the production capacity and the inventory level to different values, it is seeking to lower the production capacity. In such cases, the capital constraint on the firm is limiting and to maximise its survival probability, the firm needs to cut costs in the short term.

Note. The optimal survival strategy is not always unique. In such cases, graphs of the optimal survival strategy show the maximum and minimum order quantities and/or production capacities that maximise the probability of survival. These are labelled "maximum survival" and "minimum survival" respectively. The minimum survival levels are often zero when the capital available to the firm is sufficiently large indicating that zero inventory and zero production capacity are "optimal" decisions. The reason for this is that the constraint on the capital available to the firm is no longer limiting. The firm can effectively forego the potential profit from the next period without affecting its survival probability.

One of the most important insights of the work is to show that, most of the time, the start-up firm's optimal survival strategy should be more conservative than the established firm's 


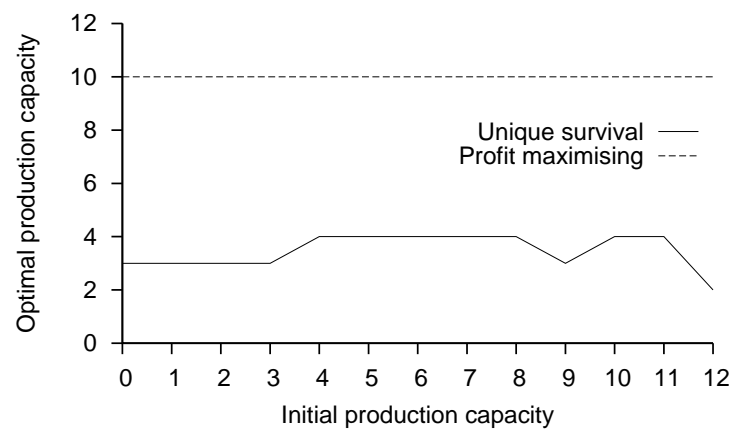

(a) Effect of initial production capacity when initial capital $X=70$ and initial inventory is 7 .

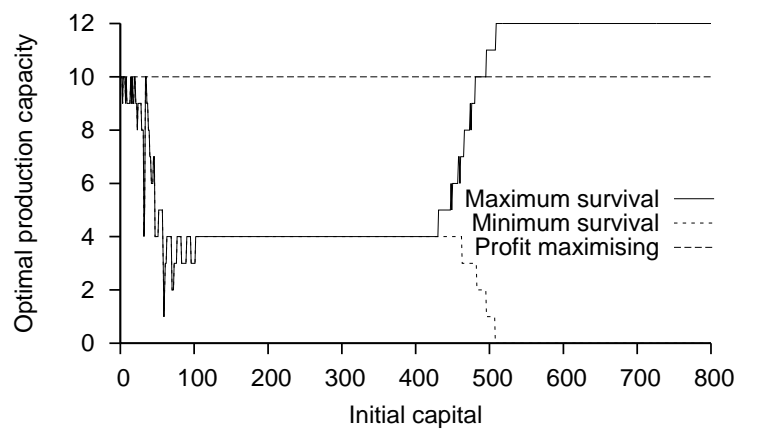

(b) Effect of initial capital when production capacity and inventory are both 10 initially.

Figure 3: Effect of the initial conditions on the production capacity decision. $S=15, C=9$, $H=4, R=5, r=3$ and $M=12$.

profit maximising one. Here conservative means to go for a lower level of production capacity. Theorem 4 gives conditions under which this result holds. The proof of Theorem 4 uses the following simple properties of the survival model.

\section{Lemma 3.}

(i) $q(i, j, x+C) \geq q(i+1, j, x)$.

(ii) $q(i, j, x+R) \geq q(i, j+1, x)$.

(iii) $q(i, j, x)$ is non-decreasing in $x$.

Proof. (i) and (ii) are consequences of the zero lead times for orders and changes to production capacity which mean that capital can be exchanged immediately for inventory or capacity. (iii) follows from the fact that in every scenario in which the firm can survive with initial capital $x$, the firm would also survive if it were to follow the same decisions with initial capital greater than $x$.

Theorem 4. Assume that $q(i, j, x)$ is continuous and differentiable with respect to $x$ in the interval $[X-H-M(C+R+r), \infty)$. The production capacity that maximises the survival probability in state $(0,0, X)$ is no greater than the production capacity that maximises the expected profit.

Proof. Theorem 2 shows that there exist optimal ordering and production capacity decisions $k, j^{\prime}$ in state $(0,0, X)$ satisfying $k=j^{\prime}$. Let $Q^{j}(d)$ be the survival probability when decisions $k=j^{\prime}=j$ are taken in state $(0,0, X)$ and demand $d$ occurs. It is easy to see from the optimality equation that $Q^{j}(d)=q(j-\min (j, d), j, X+\min (j, d) S-H-j(C+R+r))$. If $d \leq j$

$$
\begin{aligned}
& Q^{j}(d)-Q^{j+1}(d)=q(j-d, j, X+d S-H-j(C+R+r)) \\
& \quad-q(j+1-d, j+1, X+d S-H-(j+1)(C+R+r)) \\
& \geq q(j-d, j, X+d S-H-j(C+R+r)) \\
& \quad-q(j-d, j, X+d S-H-j(C+R+r)-r)) \text { by Lemma } 3 \text { (i) \& (ii) } \\
& =r q_{x}^{\prime}\left(j-d, j, \xi_{d}\right)
\end{aligned}
$$


for some $\xi_{d} \in[X+d S-H-j(C+R+r)-r, X+d S-H-j(C+R+r)]$ where $q_{x}^{\prime}$ represents the derivative of $q$ with respect to $x$. This result holds because $q$ is continuous and differentiable with respect to $x$ in this interval. If $d>j$

$$
\begin{aligned}
& Q^{j}(d)-Q^{j+1}(d)=q(0, j, X+j S-H-j(C+R+r)) \\
& \quad-q(0, j+1, X+(j+1) S-H-(j+1)(C+R+r)) \\
& \geq q(0, j, X+j S-H-j(C+R+r)) \\
& \quad-q(0, j, X+j S-H-j(C+R+r)+S-C-r) \text { by Lemma } 3 \text { (ii) } \\
& =(r+C-S) q_{x}^{\prime}\left(0, j, \xi_{j+1}\right)
\end{aligned}
$$

for some $\xi_{j+1} \in[X+j S-H-j(C+R+r), X+j S-H-j(C+R+r)+S-C-r]$.

By Lemma 3 (iii), $q(i, j, x)$ is non-decreasing in $x$, so $q_{x}^{\prime}(i, j, x) \geq 0$ in the intervals in which the $\xi_{i}$ values lie. Hence, the difference in the expected survival probabilities

$$
\sum_{d=0}^{M} p(d)\left(Q^{j}(d)-Q^{j+1}(d)\right) \geq \min _{0 \leq d \leq j+1}\left\{q_{x}^{\prime}\left(\max (0, j-d), j, \xi_{d}\right)\right\}\left(r-(S-C) \sum_{d=j+1}^{M} p(d)\right) \geq 0
$$

if $j \geq j^{*}$ by the definition of $j^{*}$ in Theorem 1 . Therefore, increasing the production capacity beyond the level which maximises the expected profit does not increase the survival probability in state $(0,0, X)$.

Remark 1. The results of numerical experiments have shown that with the exception of an interval corresponding to low levels of capital, the maximum survival probability is essentially continuous and differentiable with respect to the capital available. Hence, Theorem 4 suggests that for sufficiently large levels of capital, the production capacity that maximises the survival probability is never greater than the profit maximising production capacity. This supports one's intuition that the optimal production capacity strategy for a start-up firm should be more cautious than for a well-established firm.

\section{Application of model to a real start-up firm}

The problem that motivated this work concerned a production firm who believed capacity expansion was too risky even though it was struggling to keep up with the demand for its product. The product was essentially a component housed in a cabinet. Due to the variety of possible sizes and finishes, the firm did not keep an inventory of products. The cabinet was made from materials that were generally readily available locally and so were not routinely kept in inventory. The component had to be imported and, while the delivery time was short compared to the manufacturing time, shipments were restricted to one per week. Manufacturing was essentially employing skilled staff, which could be done quickly but on long contracts. Hence, a model of this firm would have the time period as a week and the lead times for ordering and production capacity decisions as zero. The other parameters of the model are as follows. The maximum possible production capacity is $M=12$. The demand distribution, shown in Figure 4 , is bimodal with peaks at 0 and 10 items. This models a lumpy pattern of demand in which periods tend either to be quite good or quite poor. The fixed overhead cost is $H=4$ and 


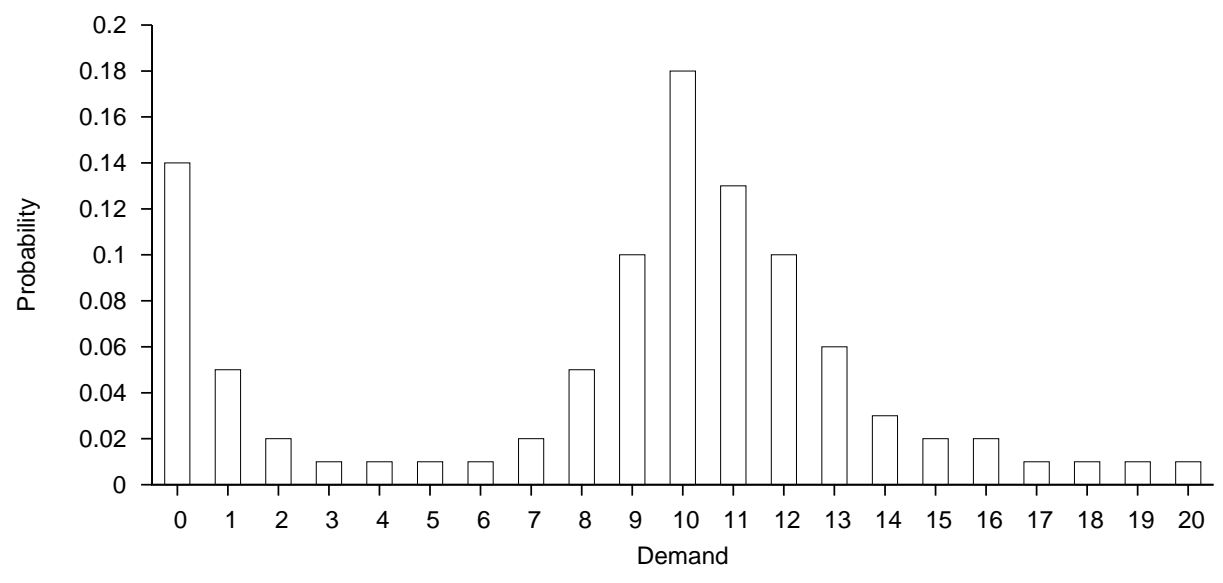

Figure 4: Weekly demand distribution for the start-up firm in the motivating example. This distribution is used in all examples.

the variable overhead cost is $r=3$ per unit of production capacity. Adjusting the production capacity costs $R=5$ per unit. The firm buys the component at unit cost $C=9$ and sells the product at unit cost $S=15$. (It is assumed that the cost of materials other than the component can be ignored.)

From Theorem 1 the profit maximising strategy is to have a production capacity of 10 and to order-up-to 10 items. Figure 5 compares the optimal survival and profit maximising policies as the capital available to the firm increases. Theorem 2 shows that for all states $(0,0, x)$ there exists an optimal survival policy which sets the order quantity and the production capacity to the same level. When the capital available is between 8 and 507 the maximum and minimum survival levels coincide, indicating a unique optimal survival policy. For high capital levels, the optimal policy is not unique, and the range of optimal actions increases until all are optimal. In that case, the firm is able to recover from any action where recover means that the difference in survival probability between the actions is smaller than the numerical accuracy to which calculations are being made. Theorem 4 suggests that, for sufficiently large capital, the survival maximising production capacity is no greater than the profit maximising production capacity. In fact, for this example, there is always an optimal survival policy which sets the production capacity to a level below the profit maximising production capacity.

As the capital available increases, the optimal survival policy can be seen to pass through 5 distinct phases.

1. The capital available is insufficient to give the firm any chance of survival regardless of the decisions taken $(0 \leq x \leq 7)$.

2. The optimal survival policy is unique and sensitive to changes in the capital available $(8 \leq x \leq 62)$. This corresponds to a situation where the policy is truly myopic and is essentially concerned about surviving the next one or two periods.

3. The optimal survival policy is unique and stable at a level that is considerably lower than the profit maximising production capacity $(63 \leq x \leq 507)$. 


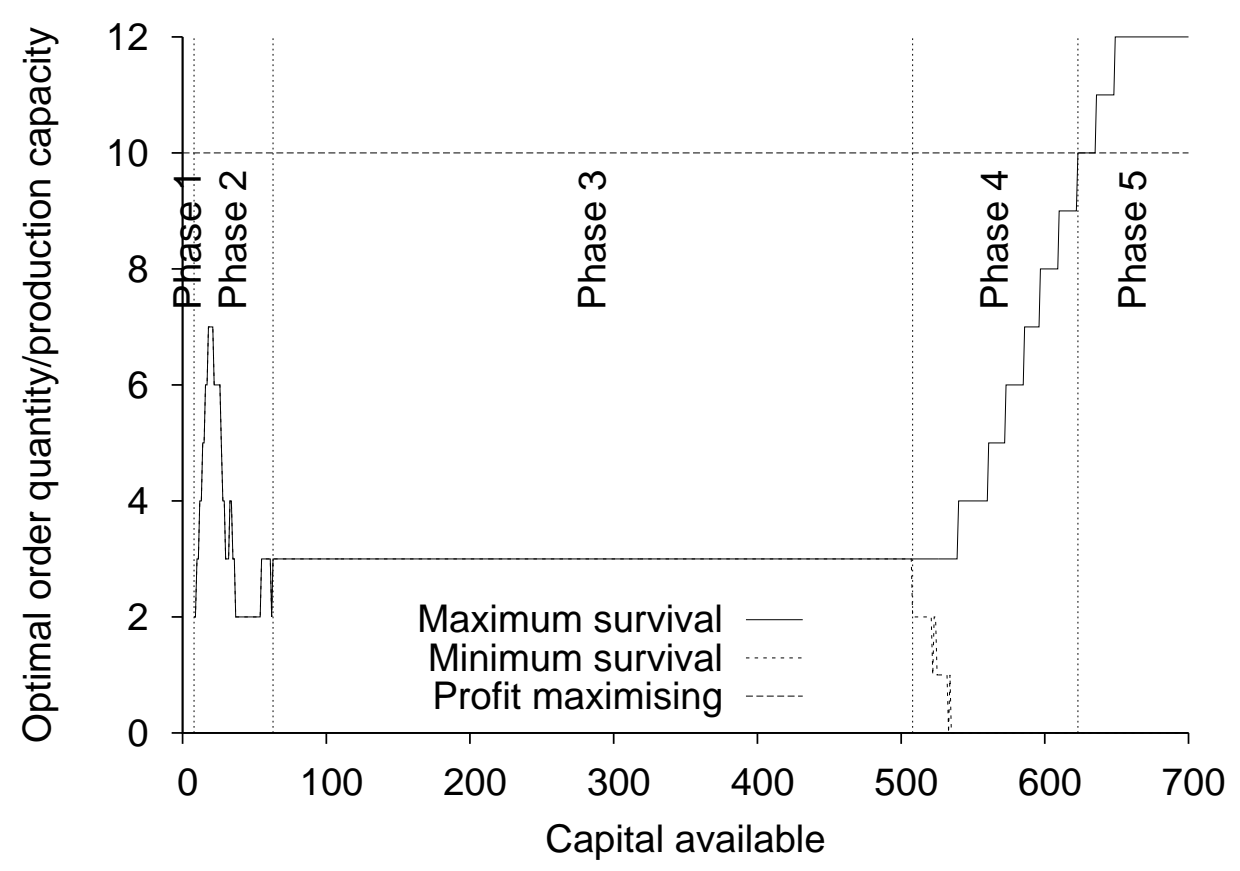

Figure 5: Comparison of the optimal survival and profit maximising policies showing the five phases of a firm's development. $S=15, C=9, H=4, R=5, r=3$ and $M=12$. Initially production capacity and inventory level are both 0 .

4. The next period's decisions are becoming less important to the chance of survival and the optimal survival policy is no longer unique $(508 \leq x \leq 622)$.

5. The range of optimal survival decisions includes the profit maximising decisions and so the firm should seek to maximise expected profit $(x \geq 623)$. This indicates the end of the start-up phase.

This behaviour is typical of optimal survival policy in all the numerical examples we have examined.

Examining the survival policy further we find that, when the production capacity is 3 and the inventory level does not exceed 3, the unique optimal actions for levels of capital between 20 and 497 are to leave the production capacity unchanged and to order-up-to 3 items. For capital above 497, these actions are still optimal but no longer unique. For capital below 20 the chance of survival is relatively low and the optimal survival policy may raise the production capacity and inventory level as high as 7 . The reason for the higher production capacity at very low capital levels in Figure 5 compared with the production capacity when capital is more than 100 is the asymmetry of the payoffs as a function of demand. With high production levels, high demand increases the capital and hence the chance of survival greatly, while low demand makes little difference in survival probability because it was so low anyway. As the capital increases the asymmetry in payoffs if production levels are high drops and, hence, more modest production levels are optimal.

When the production capacity and inventory level are both 3 , the expected profit per week is 1.76. Hence, the width of the interval over which the policy described above is the unique 
optimal survival policy is equivalent to the expected profit from a period of more than 5 years $((497-20) / 1.76$ weeks $)$. Hence, the model demonstrates that if the objective is to maximise the chance of survival, the firm should operate with a production capacity well below the profit maximising production capacity for a considerable period of time. This is a possible explanation for the seemingly overly cautious policy used by the case firm.

Figure 3 shows that the result above is not simply due to the initial conditions and the relatively high cost of adjusting the production capacity. Even when the production capacity and inventory level are initially relatively high, the optimal survival policy chooses to reduce the production capacity to a seemingly conservative level. In fact from Figure 3b, we see that even when the production capacity and the inventory level are at the profit maximising level initially, the unique optimal survival policy is to reduce the production capacity to 4 when the capital available is between 102 and 430 .

One can modify the problem by only allowing changes in production capacity at certain times rather than in each period. The extreme situation would be when the production capacity is just set once, at the beginning of the planning horizon. We have found that the optimal survival policy is just as cautious compared to the profit maximising policy when the time between production capacity decisions is varied. Figure 6a shows that, when the initial capital is between 30 and 543, a production capacity of 3 is optimal under the survival objective when the production capacity is fixed at the beginning of the planning horizon. Hence, the model demonstrates that, even if opportunities to change production capacity are infrequent, the firm should keep production capacity well below the profit maximising level to maximise its chance of survival.

Finally we investigate the effect of the profit maximising strategy on the firm's chance of survival. Figure $6 \mathrm{~b}$ compares the survival probability of the firm under the optimal survival and profit maximising policies. When the capital available is less than 8 the firm has no chance of survival regardless of the decisions taken. The maximum survival probability increases rapidly as the capital available increases from 8 to 70 . After this interval the increase in the maximum survival probability is more gradual. When the capital available is greater than 174 , the chance of the firm failing under the optimal survival policy is less than 1 in 10,000. In contrast, under the profit maximising policy, the firm needs three times as much capital to have any chance of survival. As the capital available increases from this threshold level, the increase in survival probability is relatively gradual, and it is only when the capital available exceeds 397 that the chance of the firm failing is less than 1 in 10,000.

Remark 2. These characteristics are typical of the numerical examples we have examined. It is interesting that there appears to be a threshold region for the capital available beyond which the chance of survival is very good and below which the chance of survival is slim. During the interval of rapid increase in maximum survival probability, the optimal ordering and production capacity decisions often vary greatly and, for some cost configurations, even exceed the profit maximising levels. Generally the survival probability is highly sensitive to the decisions taken in these states and adopting a profit maximising strategy would be very likely to result in the failure of the firm. These results illustrate two important insights provided by the model. Firstly the 


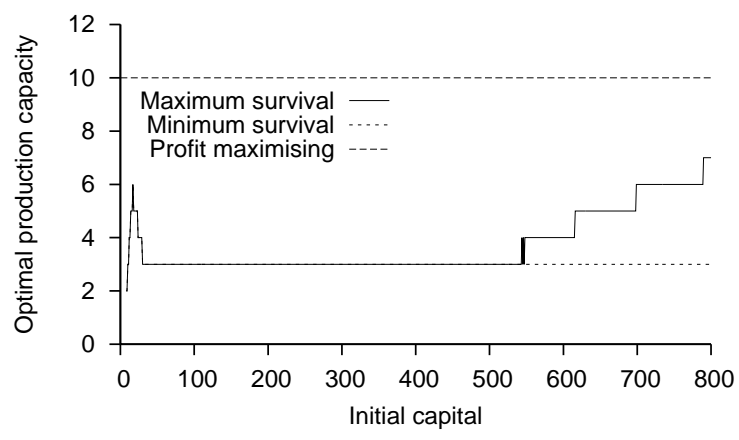

(a) Optimal policy when production capacity cannot be changed after first period.

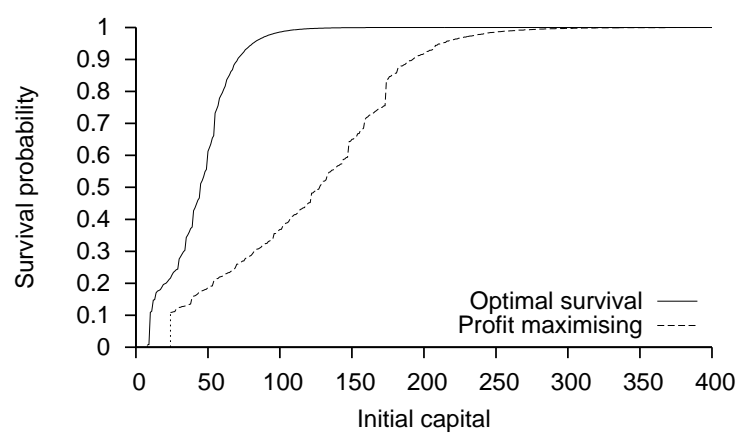

(b) Comparison of survival probability under survival and profit maximising objectives.

Figure 6: Properties of the optimal survival policy and probability under different modelling assumptions. $S=15, C=9, H=4, R=5, r=3$ and $M=12$. Initially production capacity and inventory level are both 0 .

relationship between the risk of failure and the capital invested in the firm initially. Secondly the importance of an objective that explicitly considers the chance of failure.

\section{Conclusions}

We have presented a dynamic, combined inventory and production model which determines what production capacity a manufacturing firm should invest in and how many components it should order, in the situation where the demand for the product is uncertain. This is solved under both the criterion of maximising expected profit and the criterion of maximising the probability of the firm surviving in the long term. The latter criterion has never been used in a joint production and inventory problem before nor has this model been used with the profit maximisation criterion previously. Whereas maximising expected profit is an appropriate criterion for well established firms, we have argued that maximising the probability of survival is more appropriate for start-up firms, where the decisions are very dependent on the amount of capital available.

Previous work by the authors has applied this criterion to problems involving only inventory decisions. This is the first model using this criterion to examine joint production and inventory planning. The results suggest that production capacity and inventory levels should move together as production capacity increases, but that they should be decoupled when production capacity is decreasing. These are new results.

We have proved there are sensible interactions between the production and inventory decisions under the survival objective, such as never ordering components so as to raise the inventory level above the production capacity, and if the production capacity is raised then we must raise the inventory level to this new production capacity. However, it is not the case that the inventory and production levels are always set equal to one another.

We have investigated the relationship between the profit maximising strategy and the survival maximising decisions. We describe how as the capital increases, the optimal survival strategy goes through five phases. If the capital is too low, there is no chance of survival. Immediately 
above this level, the production and inventory decisions jump to levels which are dominated by short time survival considerations and so may not be monotonic in the capital available. With more capital, the policy becomes stable but at a level considerably below the profit maximising level. At some point there is sufficient capital available so that the next decision is not vital and so the optimal survival policy is not unique and eventually in the fifth phase, the profit maximising decisions are also optimal for the survival probability model. At this point the firm should change to the profit maximising criterion.

Thus, the paper does seem to explain why in the motivating example the firm believed it was better to operate for some time with a production level which was significantly below the profit maximising one. It was subjectively recognising that survival was its most important objective, and was in the third phase of the optimal survival policy outlined above.

We have also investigated the extent to which our findings depend on the assumptions of an infinite horizon and regular opportunities to adjust production capacity. Perhaps surprisingly, we find that the optimal survival and profit maximising policies are fairly robust to changes in the length of the planning horizon and the time between production decisions. Importantly for our explanation of the behaviour of the firm in the motivating example, the five phases of the optimal survival policy can still be detected in finite horizon models with infrequent production decisions.

The model described could be reinterpreted as a marketing-production problem where one is interested in the mix of spending on advertising and on component inventory levels. One can think of production capacity as a limitation on the potential demand that can be turned into sales. So if one goes from production level $j$ to production level $j^{\prime}$ one is paying a cost $R\left|j-j^{\prime}\right|$ to increase the potential level of sales and an amount $r j^{\prime}$ to sustain the sales at that potential maximum level.

One could think of advertising as doing the same sort of thing. The demand distribution in the model represents the potential demand if the whole population were aware of the product. In order to make $j$ customers aware of the product, and hence have a potential maximum of $j$ sales, one has to sustain an advertising spend of $r j$. If one wants to increase the potential maximum sales level, one has to develop further advertising at a cost of $R\left|j-j^{\prime}\right|$, as well as then keeping the advertising spend at $r j^{\prime}$. One might quibble about the extra cost $R$ being involved if one wants to lower the advertising spend, but one could envisage penalty clauses in the contracts with the advertising media which would require payment if the advertising is cancelled. This is a simple model of the way advertising interacts with total sales, but it does allow one to investigate the impact that marketing may have on the survival potential of start-up firms. Production capacity is strongly related to the manpower available as well as the equipment that the firm has. So one could also think of this model as one on manpower planning, with the costs of changing levels of production corresponding to hiring and redundancy costs.

One could develop more realistic models of the situation faced by small production companies by allowing the demand to be non-stationary (reflecting the growth phase of a firm) or by making the demand a function of price. These are worth further investigation, but will mean some of the clarity in what is already a complex model will be lost. 
We believe that this paper does contribute to an understanding of the operations management for start-up firms, by looking at the coordination needed between production and inventory decisions, and it does suggest ways of investigating what is the best strategic mix of investment in production capacity and component availability.

\section{Acknowledgements}

We are honoured to be part of this special issue in memory of Doug White. One of us (LT) was privileged to learn about Markov decision processes directly from Doug and his friendship, kindness and research leadership will always be remembered. Doug introduced one of the earliest production capacity planning problems in his book on dynamic programming White (White, 1969) and was always interested in the application of Markov decision processes to real problems (White, 1985, 1988, 1993). We are all grateful for the influence Doug had on research in this and many other areas of decision theory and operational research.

\section{Appendix}

Proof of Theorem 1. It is easy to verify by substitution that the given values for $g$ and $v(i, j)$ satisfy the optimality equation for the stated policies. The policy improvement step of policy iteration involves finding $k$ and $j^{\prime}$ to maximise the right hand side of the optimality equations for given value of $v(i, j)$.

$$
\begin{aligned}
& \max _{k, j^{\prime}}\left\{\sum_{d=0}^{M} p(d)\left(\min \left(i+k, j^{\prime}, d\right) S-k C-\left|j-j^{\prime}\right| R-H-j^{\prime} r+\left(i+k-\min \left(i+k, j^{\prime}, d\right)\right) C-\left|j^{\prime}-j^{*}\right| R\right)\right\} \\
& =i C-H+\max _{j^{\prime}}\left\{-\left|j-j^{\prime}\right| R-\left|j^{\prime}-j^{*}\right| R-j^{\prime} r+(S-C) \max _{k}\left\{\sum_{d=0}^{M} p(d) \min \left(i+k, j^{\prime}, d\right)\right\}\right\}
\end{aligned}
$$

For $i+k<j^{\prime}, \sum_{d=0}^{M} p(d) \min \left(i+k, j^{\prime}, d\right)$ increases with $k$ while, for $i+k \geq j^{\prime}$, it is independent of $k$. Hence, $k=\max \left(0, j^{\prime}-i\right)$ is an optimal order quantity.

With this choice of $k, i+k=i+\max \left(0, j^{\prime}-i\right)=\max \left(i, j^{\prime}\right) \geq j^{\prime}$. Hence, $\min \left(i+k, j^{\prime}, d\right)=$ $\min \left(j^{\prime}, d\right)$. The policy improvement step is completed by finding $j^{\prime}$ in the following expression.

$$
\begin{aligned}
& \max _{j^{\prime}}\left\{-\left|j-j^{\prime}\right| R-\left|j^{\prime}-j^{*}\right| R-j^{\prime} r+(S-C) \sum_{d=0}^{M} p(d) \min \left(j^{\prime}, d\right)\right\} \\
& \quad=\max _{j^{\prime}}\left\{-\left(\left|j-j^{\prime}\right| R+\left|j^{\prime}-j^{*}\right|\right) R-j^{\prime} r+(S-C) \sum_{d \leq j^{\prime}} p(d) d+j^{\prime}(S-C) \sum_{d>j^{\prime}} p(d)\right\}
\end{aligned}
$$

Suppose $j^{\prime} \neq j^{*}$. Moving $j^{\prime}$ one unit in the direction of $j^{*}$ reduces $\left|j^{\prime}-j^{*}\right|$ by one and increases $\left|j-j^{\prime}\right|$ by at most one. Therefore the first term in the maximisation above is either unchanged or increased by $R$. 
If $j^{\prime}<j^{*}$, increasing $j^{\prime}$ by one unit changes the value of the other terms in the maximisation by:

$$
\begin{aligned}
& -r+(S-C) p\left(j^{\prime}+1\right)\left(j^{\prime}+1\right)-(S-C) j^{\prime} p\left(j^{\prime}+1\right)+(S-C) \sum_{d>j^{\prime}+1} p(d) \\
& =-r+(S-C) \sum_{d>j^{\prime}} p(d) \geq 0 \text { by definition of } j^{*} .
\end{aligned}
$$

Hence, increasing the value of $j^{\prime}$ by one unit in the direction of $j^{*}$ does not reduce the value of the expression in the maximisation and there exists an optimal choice for $j^{\prime} \geq j^{*}$.

If $j^{\prime}>j^{*}$, decreasing $j^{\prime}$ by one unit changes the value of the final three terms in the maximisation by:

$$
\begin{aligned}
r & -(S-C) p\left(j^{\prime}\right) j^{\prime}+(S-C)\left(j^{\prime}-1\right) p\left(j^{\prime}\right)-(S-C) \sum_{d>j^{\prime}} p(d) \\
& =r-(S-C) \sum_{d>j^{\prime}-1} p(d)>0 \text { by definition of } j^{*} .
\end{aligned}
$$

Hence, decreasing the value of $j^{\prime}$ by one unit in the direction of $j^{*}$ increases the value of the expression in the maximisation and the optimal value of $j^{\prime}$ can be at most $j^{*}$. It follows that $j^{\prime}=j^{*}$ is an optimal production level.

The maximising actions, $k=\max \left(0, j^{*}-i\right)$ and $j^{\prime}=j^{*}$, correspond to the stated policy, so the policy improvement step has failed to find a better policy. It follows that this policy is optimal.

Proof of Theorem 2. For state $(i, j, x)$, let $k$ be the smallest optimal order quantity and $j^{\prime}$ be the smallest optimal production capacity for order quantity $k$.

(i) Suppose that $k>0$ and $i+k>j^{\prime}$. Let $\delta=\min \left(k, i+k-j^{\prime}\right)$. The decision to order $\delta$ fewer items in state $(i, j, x)$ is feasible but not optimal, so $q(i, j, x)$

$$
\begin{aligned}
& >\sum_{d=0}^{M} p(d) q\left(i+k-\delta-\min \left(d, j^{\prime}\right), j^{\prime},\right. \\
& \left.\quad x+\min \left(d, j^{\prime}\right) S-(k-\delta) C-\left|j-j^{\prime}\right| R-H-j^{\prime} r\right) \\
& \geq \sum_{d=0}^{M} p(d) q\left(i+k-\min \left(d, j^{\prime}\right), j^{\prime}, x+\min \left(d, j^{\prime}\right) S-k C-\left|j-j^{\prime}\right| R-H-j^{\prime} r\right) \\
& \quad \text { by Lemma } 3 \text { (i) } \\
& =q(i, j, x)
\end{aligned}
$$

This is a contradiction, so either $k=0$ or $i+k \leq j^{\prime}$.

(ii) Suppose that $j^{\prime}>j$ and $i+k<j^{\prime}$. Let $\delta=\min \left(j^{\prime}-j, j^{\prime}-i-k\right)$. The decision to set the production capacity to $j^{\prime}-\delta$ units (with order quantity $k$ ) in state $(i, j, x)$ is feasible but not optimal, so $q(i, j, x)$

$$
>\sum_{d=0}^{M} p(d) q\left(i+k-\min (d, i+k), j^{\prime}-\delta,\right.
$$




$$
\begin{aligned}
& \left.x+\min (d, i+k) S-k C-\left(j^{\prime}-\delta-j\right) R-H-\left(j^{\prime}-\delta\right) r\right) \\
& \text { since } j^{\prime}-\delta \geq i+k \text { and } j^{\prime}-\delta \geq j \\
\geq & \sum_{d=0}^{M} p(d) q\left(i+k-\min (d, i+k), j^{\prime}\right. \\
& \left.x+\min (d, i+k) S-k C-\left(j^{\prime}-j\right) R-H-\left(j^{\prime}-\delta\right) r\right) \text { by Lemma } 3 \text { (ii) } \\
\geq & \sum_{d=0}^{M} p(d) q\left(i+k-\min (d, i+k), j^{\prime},\right. \\
= & q(i, j, x)
\end{aligned}
$$

This is a contradiction, so either $j^{\prime} \leq j$ or $i+k \geq j^{\prime}$.

\section{References}

Angelus, A., Porteus, E. L., 2002. Simultaneous capacity and production management of shortlife-cycle, produce-to-stock goods under stochastic demand. Management Science 48 (3), 399413.

Archibald, T. W., Thomas, L. C., Betts, J. M., Johnston, R. B., 2002. Should start-up companies be cautious? Inventory policies which maximise survival probabilities. Management Science 48 (9), 1161-1174.

Archibald, T. W., Thomas, L. C., Possani, E., 2007. Keep or return? Managing ordeing and return policies in start-up companies. European Journal of Operational Research 179 (1), $97-113$.

Babich, V., Sobel, M. J., 2004. Pre-IPO operational and financial decisions. Management Science $50(7), 935-948$.

Betts, J., Johnston, R. B., 2001. Just-in-time replacement decisions for assembly manufacturing with investor-supplied finance. Journal of the Operational Research Society 52 (7), 750-761.

Buzacott, J. A., Zhang, R. Q., 2004. Inventory management with asset-based pricing. Management Science 50 (9), 1274-1292.

Cantamessa, M., Valentini, C., 2000. Planning and managing manufacturing capacity when demand is subject to diffusion effects. International Journal of Production Economics 66 (3), $227-240$.

Chan, L. M. A., Simchi-Levi, D., Swann, J., 2006. Pricing, production, and inventory policies for manufacturing with stochastic demand and discretionary sales. Manufacturing and Service Operations Management 8 (2), 149-168. 
Çınar, E., Güllü, R., 2012. An inventory model with capacity flexibility in the existence of advance capacity information. Decision Support Systems 53 (2), 320-330.

Dellaert, N., de Kok, T., 2004. Integrating resource and production decisions in a simple multistage assembly system. International Journal of Production Economics 90 (3), 281-294.

Jammernegg, W., Reiner, G., 2007. Performance improvement of supply chain processes by coordinated inventory and capacity management. International Journal of Production Economics 108 (1-2), 183-190.

Li, L., Shubik, M., Sobel, M. J., 2013. Control of dividends, capital subscriptions, and physical inventories. Management Science 59 (5), 1107-1124.

Mincsovics, G., Tan, T., Alp, O., 2009. Integrated capacity and inventory management with capacity acquisition lead times. European Journal of Operational Research 196 (3), 949-958.

Possani, E., Thomas, L. C., Archibald, T. W., 2003. Loans, ordering and shortage costs in startups: a dynamic stochastic decision approach. Journal of the Operational Research Society $54(5), 539-548$.

Puterman, M. L., 1994. Markov decision processes: Discrete stochastic dynamic programming. John Wiley, New York.

Silver, E. A., Pyke, D. F., Peterson, R., 1998. Inventory Management and Production Planning and Scheduling. John Wiley, New York.

Sobel, M. J., 1969. Production smoothing with stochastic demand I: Finite horizon case. Management Science 16 (3), 195-207.

Sobel, M. J., 1970. Employment smoothing (capital-accumulation) with production for stochastic demand. Management Science 16 (5), 340-349.

Sobel, M. J., 1971. Production smoothing with stochastic demand II: Infinite horizon case. Management Science 17 (11), 724-735.

Statistic Brain, 2013. Startup business failure rate by industry. URL: www.statisticbrain.com/startup-failure-by-industry, Accessed: 16 August 2013.

Swinney, R., Cachon, G. P., Netessine, S., 2011. Capacity investment timing by start-ups and established firms in new markets. Management Science 57 (4), 763-777.

Tanrisever, F., Erzurumlu, S. S., Joglekar, N., 2012. Production, process investment, and the survival of debt-financed startup firms. Production and Operations Management 21 (4), 637652.

Terwiesch, C., Bohn, R. E., 2001. Learning and process improvement during production rampup. International Journal of Production Economics 70 (1), 1-19. 
van Mieghem, J. A., 2003. Capacity management, investment, and hedging: Review and recent developments. Manufacturing and Service Operations Management 5 (4), 269-302.

White, D. J., 1969. Dynamic Programming. Oliver and Boyd, Edinburgh.

White, D. J., 1985. Real applications of Markov decision processes. Interfaces 15 (6), 73-83.

White, D. J., 1988. Further real applications of Markov decision processes. Interfaces 18 (5), $55-61$.

White, D. J., 1993. A survey of applications of Markov decision processes. Journal of the Operational Research Society 44 (11), 1073-1096. 